\title{
Anti-neuroinflammatory Effect of Plantago Major var. Japonica in BV-2 Microglial Cells
}

\author{
Hyun Kang ${ }^{\dagger}$ \\ Department of Medical Laboratory Science, College of Health Science, \\ Dankook University, Chungnam 31116, Korea
}

To evaluate the protective effects of Plantago Major extract (PME) in stimulated BV-2 microglial cells and its anti-oxidant properties, cell viability assessment was performed by 3-(4, 5-dimethylthiazol-2-yl)-2, 5-diphenyltetrazolium bromide (MTT) assay. Lipopolysaccharide (LPS) was used to activate BV-2 microglia. Nitric oxide (NO) levels were measured using Griess assay. Tumor necrosis factor-alpha (TNF- $\alpha$ ) production was evaluated by enzyme-linked immunosorbent assay (ELISA). Antioxidant properties were evaluated by 1, 1-diphenyl-2-picryl-hydrazyl (DPPH) radical scavenging assay. LPS-activated excessive release of NO in BV-2 cells was significantly inhibited by $\mathrm{PME}(P<0.001$ at $100 \mu \mathrm{g} / \mathrm{mL})$. PME also scavenged DPPH radicals in a dose-dependent manner $(P<0.05$ at $10 \mu \mathrm{g} / \mathrm{mL}$ and $P<0.001$ at $20 \sim 200 \mu \mathrm{g} / \mathrm{mL}$ ). These results indicate that PME attenuated neuroinflammatory responses in LPS-activated BV-2 microglia by inhibiting excessive production of pro-inflammatory mediators such as NO and TNF- $\alpha$. The anti-neuroinflammatory potential of PME may be related to its strong antioxidant properties.

Key Words: Plantago Major, DPPH radicals, Anti-oxidant, Neuroinflammation, BV-2 microglia, Nitric oxide

Microglia cells are immune cells in the central nervous system (CNS) that able to produce several inflammatory mediators in response to stressors. Activated microglia play a critical role in the neuroinflammatory processes by releasing toxic mediators including nitric oxide (NO), inducible NO synthase (iNOS), interleukins (IL), tumor necrosis factoralpha (TNF- $\alpha$ ), and free radicals (Matsumoto et al., 1992; Gao et al., 2002). Activated microglia and the released inflammatory mediators may lead to several neurodegenerative diseases including multiple sclerosis (MS), Parkinson's disease (PD), Alzheimer's disease (AD) and Huntington's disease (Chiang et al., 2003). It is well known that microglia can be activated by lipopolysaccharide (LPS) and is recognized to be a useful in vitro tool for studying neuroinflammatory mechanisms (Kim et al., 2004). LPS activated BV-2 microglia cells enhance the production of immune-related cytotoxic factors and pro-inflammatory cytokines (Kim et al., 2004; Park et al., 2013). Thus, the control of microglial activation has been suggested as a promising therapeutic target in combating neuroinflammatory-mediated neurodegenerative diseases. Plantago L. (Plantaginaceae) is a genus widely distributed all over the world, and more than about 300 species have been reported (Beara et al., 2009). Plantago Major is an endemic species found in South Eastern Asia including Korea peninsula. It is used traditionally and commercially available (Velasco-Lezama et al., 2006). Plantago

* Received: September 26, 2017 / Revised: November 21, 2017 / Accepted: November 21, 2017

†Corresponding author: Hyun Kang. Department of Medical Laboratory Science, College of Health Science, Dankook University, Cheonan-si, Chungnam 31116, Korea.

Tel: +82-41-550-3015, Fax: +82-41-559-7934, e-mail: hkang@dankook.ac.kr

(C) The Korean Society for Biomedical Laboratory Sciences. All rights reserved.

(c) This is an Open Access article distributed under the terms of the Creative Commons Attribution Non-Commercial License (http://creativecommons.org/licenses/by-nc/3.0/) which permits unrestricted non-commercial use, distribution, and reproduction in any medium, provided the original work is properly cited. 
Major has been reported to have anticancer and antiinflammatory properties. However, its anti-neuroinflammatory mechanisms have not been fully elucidated and till date the effect of Plantago Major leaf extract on neuroinflammation and LPS-activated microglial neurotoxicity has not been documented. The aim of this study was to investigate whether Plantago Major exhibits protective effects on LPS-activated neuroinflammatory processes in BV-2 microglial cells.

To obtain the Plantago Major leaf extract (PME), fresh leaves were washed to remove debris and air-dried for two days. The dried leaves were then ground to powder using an electric blender (Model 4250, Braun Germany). The powdered leaf was extracted with three volumes of $80 \%$ ethanol with mixing at room temperature for $1 \mathrm{~h}$. The extract was filtered and lyophilized to obtain ethanol extract concentrate. The EtOH extract of PME obtained was re-suspended in water: $\mathrm{EtOH}(9: 1, \mathrm{v} / \mathrm{v})$ and partitioned in turn via n-hexane, chloroform, ethyl acetate (EA) and n-butanol solvents. Since EA fraction of PME showed potent antioxidant effect in our preliminary evaluation, further studies on anti-neuroinflammatory effects in LPS-stimulated BV-2 microglial cells was investigated using PME extract. The PME extract was dissolved in sterile distilled water, filtered on $0.22 \mu \mathrm{m}$ filters and stored at $-20^{\circ} \mathrm{C}$. All reagents used in this study were the highest grade commercially available. The anti-oxidant activity of the PME extract was determined using the stable radical 2, 2-diphenyl-1-picrylhydrazyl (DPPH, Sigma-Aldrich, St. Louis, MO, USA). The radical scavenging capacity was evaluated by employing a reaction mixture constituted by aliquots of the PME extract and a DPPH methanolic solution as described previously (Nanjo et al., 1996). BV-2 microglia cells were cultured at $37^{\circ} \mathrm{C}$ in $5 \% \mathrm{CO}_{2}$ in DMEM (Invitrogen, Carlsbad, CA, USA) supplemented with 5\% FBS (Hyclone, Logan, UT, USA) and antibiotics (Invitrogen). In all experiments, cells were pre-treated with PME extract at indicated concentrations $10 \sim 100 \mu \mathrm{g} / \mathrm{mL}$ ) for $1 \mathrm{~h}$ before the addition of LPS ( $5 \mu \mathrm{g} / \mathrm{mL}$, Sigma-Aldrich, St Louis, MO, USA) in serum free DMEM. An equal volume of sterile water was added to all control treatments. For viability assay, 3-(4, 5-dimethylthiazol-2-yl)-2, 5-diphenyl-tetrazolium bromide (MTT, Sigma-Aldrich, St. Louis, MO, USA) assay was used as described previously (Kim et al., 2011). Production of NO was assayed by measuring the levels of nitrite in the culture supernatant using colorimetric assay with Griess reagent (Kim et al., 2011). BV-2 microglia cells $\left(1 \times 10^{5}\right.$ cells/well) were cultured on 96 well plates and treated with the PME extract at indicated concentrations for $1 \mathrm{~h}$ and stimulated with LPS $(5 \mu \mathrm{g} / \mathrm{mL})$. At $4 \mathrm{~h}$ post-LPS treatment, the cells were collected and the supernatants were evaluated for TNF- $\alpha$ level using a murine TNF- $\alpha$ ELISA kit from BD Biosciences (San Jose, CA, USA) according to the manufacturer's instructions. All data are presented as mean \pm SEM of at least three independent experiments. Statistical analysis was performed with SAS statistical software (SAS Institute, Cray, NC, USA) using one-way analysis of variance, followed by Dunnett's multiple range tests. $P<0.05$ was considered statistically significant.

Previous studies have indicated a variety of beneficial pharmacological effects of $P$. major such as anticancer (Chiang et al., 2003), antioxidant, (Beara et al., 2009; Kolak et al., 2011) and immunomodulatory (Gomez-Flores et al., 2000). Concerning its anti-inflammatory activity, several in vivo experiments were carried out to prove this property (Núñez Guillén et al., 1997).

Anti-neuroinflammatory activity of $P$. major through modulation of inflammatory cytokines, however, has not been well characterized. Neuroinflammation is characterized by the activation of microglia and expression of major inflammatory mediators in the CNS. The importance of early therapeutic interference to inhibit microglial activation would be an effective. Mounting evidence has implicated the pathogenesis of several human diseases including neurodegenerative disorders related to increased oxidative stress (Finkel and Holbrook, 2000). Increased reactive oxygen species (ROS) production can regulate the expression of diverse inflammatory mediators during brain injury. Elevated levels of several pro-inflammatory factors including toxic free radicles in the CNS have been detected in patients with neurodegenerative diseases (Uttara et al., 2009). Therefore, inhibition by antioxidants and radical scavengers can reduce neuroinflammation.

It is well known that DPPH radical assay is one of the widely used methods for evaluating the free radical scavenging activities of several antioxidants in a relatively short 
period of time (Nanjo et al., 1996). Several studies have reported that antioxidants play important roles in the prevention of aging and age-related diseases (Uttara et al., 2009). As shown in Fig. 1, PME exhibited significant DPPH radical scavenging activity in a concentration-dependent manner showing a maximum effect at $100 \mu \mathrm{g} / \mathrm{mL}(P<0.001)$. Treatment with PME extract at indicated concentrations $(10 \sim 100$ $\mu \mathrm{g} / \mathrm{mL}$ ) did not affect the overall cell viability nor did they exhibit any cytotoxicity on BV2 microglia cells. Although

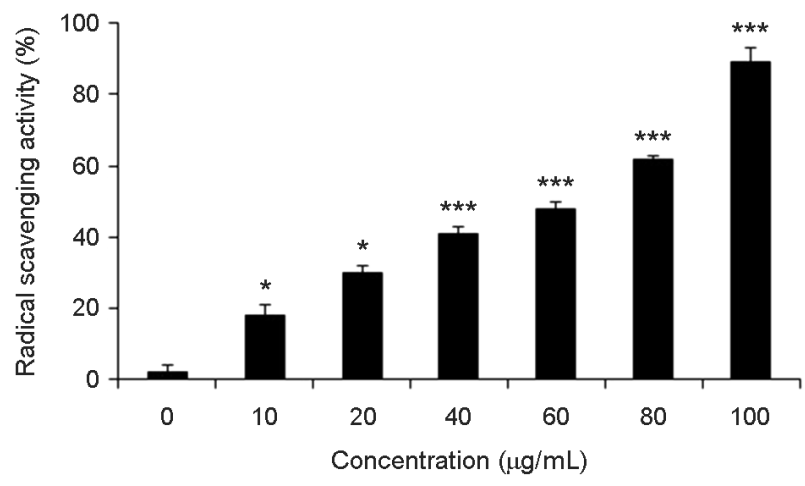

Fig. 1. Effect of PME extract on DPPH radical scavenging activity. The capacities to scavenge DPPH radicals by different concentrations $(10,20,40,60,80$ and $100 \mu \mathrm{g} / \mathrm{mL}$ ) of PME extract (Plantago Major-ethylacetate). Data are presented as mean \pm SEM $(\mathrm{n}=3)$ for three independent experiments; ${ }^{*} P<0.05$, and ${ }^{* * *} P<0.001$, compared with control group.

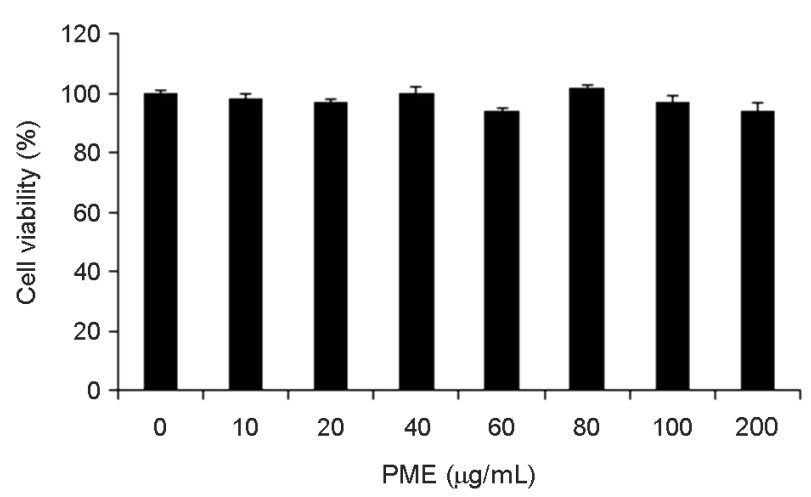

Fig. 2. Effects of PME extract on the viability of BV-2 microglial cells. Viability in PME extract-treated cells was determined using MTT assay in the presence or absence of LPS $(5 \mu \mathrm{g} / \mathrm{mL})$. The results are depicted as percentage of control samples. Data are presented as the mean \pm S.E.M. $(n=3)$ for three independent experiments. No significant when difference compared with control group (PME: Plantago Major-ethylacetate). not significant, PME at $200 \mu \mathrm{g} / \mathrm{mL}$ concentration showed moderate signs of cytotoxicity (Fig. 2). Therefore $100 \mu \mathrm{g} / \mathrm{mL}$ of PME was utilized as maximum concentration for further experiments and activities. It was well documented that one

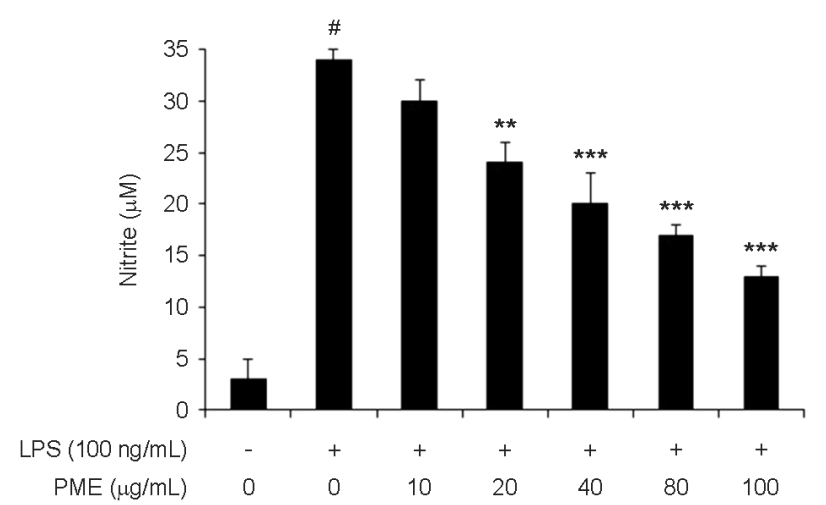

Fig. 3. Effect of PME extract on NO Production in LPS stimulated BV-2 microglial cells. BV-2 cells were treated with PME extract at various concentrations $(20,40,80$ and $100 \mu \mathrm{g} / \mathrm{mL})$ with or without LPS $(5 \mu \mathrm{g} / \mathrm{mL})$ for $4 \mathrm{~h}$. The nitrite in the culture supernatant was evaluated using Griess reagent. Data are presented as the mean \pm S.E.M. $(\mathrm{n}=3)$ for three independent experiments. $\# P<0.001$, when compared with control group. $* * P<0.01$ and $* * * P<0.001$, when compared with LPS alone treated group by Student $t$-test (PME: Plantago Major-ethylacetate, LPS: Lipopolysaccharide).

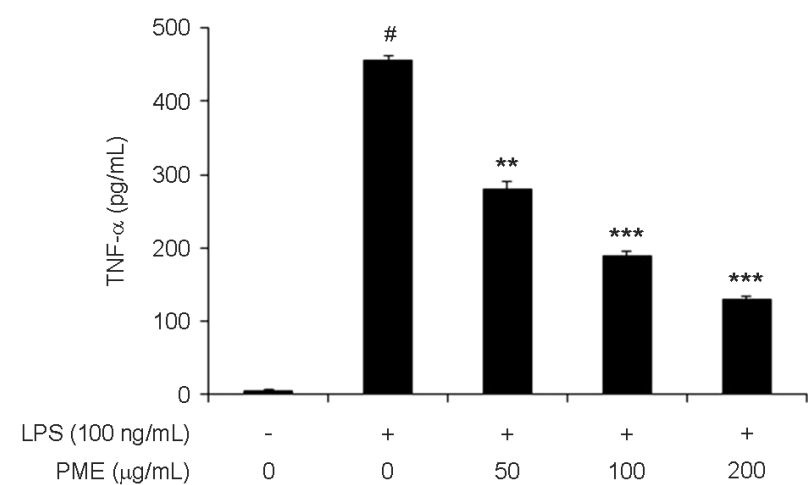

Fig. 4. Effect of PME extract on TNF- $\alpha$ production in LPSstimulated BV-2 microglial cells. Suppression of pro-inflammatory cytokine TNF- $\alpha$ expression by PME extract was measured with ELISA test. BV-2 cells were treated with PME extract at 50 and $100 \mu \mathrm{g} / \mathrm{mL}$ with or without LPS $(5 \mu \mathrm{g} / \mathrm{mL})$ for $4 \mathrm{~h}$. The TNF- $\alpha$ in the culture supernatant was evaluated using a murine TNF- $\alpha$ ELISA kit. Data are presented as the mean \pm S.E.M. $(n=3)$ for three independent experiments. $\# P<0.001$, when compared with control group. $* * P<0.01$ and $* * * P<0.001$, when compared with LPS alone group by Student $t$-test (PME: Plantago Major-ethylacetate, LPS: Lipopolysaccharide, TNF- $\alpha$ : Tumor necrosis factor- $\alpha$ ). 
of the major pro-inflammatory mediators released by activated microglia is $\mathrm{NO}$, an uncharged lipophilic molecule that is toxic to neurons. Elevated levels of pro-inflammatory cytokines and mediators are also up-regulated in brain once microglial cells are activated (Gao et al., 2002; Merill et al., 2009). Cells treated with LPS ( $5 \mu \mathrm{g} / \mathrm{mL})$ alone significantly increased in NO levels $(P<0.001)$. Pre-treatment with PME extract at indicated concentrations significantly and dosedependently suppressed the excessive release of NO in BV-2 cells in a (Fig. 3). Although PME at $10 \mu \mathrm{g} / \mathrm{mL}$ concentration did not influence the NO release, 20, 40, 60, 80 and $100 \mu \mathrm{g} /$ $\mathrm{mL}$ concentrations showed significant effects. The maximum effect was observed at a concentration of $100 \mu \mathrm{g} / \mathrm{mL}(P<$ 0.001). As shown in Fig. 4, TNF- $\alpha$ levels increased significantly after LPS treatment $(5 \mu \mathrm{g} / \mathrm{mL})$ when compared to those in untreated cells $(P<0.001)$. However, PME extract significantly inhibited TNF- $\alpha$ production in a concentrationdependent manner in LPS-stimulated BV-2 cells $(P<0.01$ at $50 \mu \mathrm{g} / \mathrm{mL}$ and $P<0.001$ at $100 \mu \mathrm{g} / \mathrm{mL}$, respectively). Therefore, inhibition of cytokine production in activatedmicroglia might serve as a key mechanism in the control of neuroinflammatory responses in neurodegeneration.

In the present study, PME extract significantly inhibited the LPS-stimulated increase in NO production and suppressed the iNOS protein expression in LPS-stimulated BV-2 cells. Activated microglial cells are known to release several proinflammatory cytokines including TNF- $\alpha$ which may not only amplify the inflammatory cascade, but also cause inflammatory injury (Perry et al., 2001; Frankola et al., 2011). Therefore, we investigated whether PME extract had an effect on the production of TNF- $\alpha$ in LPS activated BV-2 microglial cells. This indicated that the leaves could be included in the preparation of $P$. major derived herbal medicines. Our results indicate that PME extract significantly suppressed the production of TNF- $\alpha$ in the LPSactivated BV-2 cells. This study revealed that $P$. major extract attenuated the neuroinflammatory processes in LPSinduced BV-2 microglial cells. The anti-neuroinflammatory effects of PME extract might be attributed it its regulatory actions on proinflammatory cytokine such as TNF- $\alpha$ and its strong antioxidant effects. Based on our results PME extract might be developed as a promising candidate for the treat- ment of neuroinflammation-mediated neurological disorders.

\section{ACKNOWLEDGEMENTS}

None.

\section{CONFLICT OF INTEREST}

The authors declare that there is no conflict of interests regarding the publication this articles.

\section{REFERENCES}

Bruno R, Dugan C, Smyth J, DiNatale D, Koo S. Green tea extract protects leptin-deficient, spontaneously obese mice from hepatic steatosis and injury. American Society for Nutrition. 2008. 21: 323-331.

Beara IN, Lesjak MM, Jovin ED, Balog KJ, Anackov GT, Orcic DZ. Plantain (Plantago L.) species as novel sources of flavonoid antioxidants. Journal of Agricultural and Food Chemistry. 2009. 57: 9268-9273.

Chiang LC, Chiang W, Chang MY, Lin CC. In vitro cytotoxic, antiviral and immunomodulatory effects of Plantago Major and Plantago asiatica. The American Journal of Chinese Medicine. 2003. 31: 225-234.

Finkel T, Holbrook NJ. Oxidants. oxidative stress and the biology of ageing. Nature. 2000. 408: 239-247.

Frankola KA, Greig NH, Luo W, Tweedie D. Targeting TNF-alpha to elucidate and ameliorate neuroinflammation in neurodegenerative diseases. CNS \& Neurological Disorders Drug Targets. 2011. 10: 391-403.

Gao HM, Jiang J, Wilson B, Zhang W, Hong JS, Liu B. Microglial activation-mediated delayed and progressive degeneration of rat nigral dopaminergic neurons: relevance to Parkinson's disease. Journal of Neurochemistry. 2002. 81: 1285-1297.

Gomez-Flores R, Calderon CL, Scheibel LW, Tamez-Guerra P, Rodriguez-Padilla C, Tamez-Guerra R. Immuno-enhancing properties of Plantago Major leaf extract. Phytotherapy Research. 2000. 14: 617-622.

Kim BW, Koppula S, Kim IS, Lim HW. Antineuroinflammatory activity of Kamebakaurin from Isodon japonicus via inhibition of c-Jun NH-terminal kinase and p38 mitogen-activated protein kinase pathway in activated microglial cells. Journal of Pharmacological Sciences. 2011. 116: 296-308.

Kim SH, Smith CJ, Van Eldik LJ. Importance of MAPK pathways for microglial pro-inflammatory cytokine IL-1 $\beta$ production. 
Neurobiology of Aging. 2004. 25: 431-439.

Kolak U, Boğa M, Uruşak EA, Ulubelen A. Constituents of Plantago Major subsp. intermedia with antioxidant and anticholinesterase capacities. Turkish Journal of Chemistry. 2011. 35: 637-645.

Nanjo F, Goto K, Seto R, Suzuki M. Scavenging effects of tea catechins and their derivatives on 1,1-diphenyl-2-picrylhydrazyl radical. Free Radical Biology and Medicine. 1996. 21: 895 -902 .

Núñez Guillén ME, da Silva Emim JA, Souccar C, Lapa AJ. Analgesic and anti-inflammatory activities of the aqueous extract of Plantago Major L. Pharmaceutical Biology. 1997. 35: 99-104.

Matsumoto Y, Ohmori K, Fujiwara M. Immune regulation by brain cells in the central nervous system: microglia but not astrocytes present myelin basic protein to encephalitogenic $\mathrm{T}$ cells under in vivo mimicking conditions. Immunology. 1992. 76: 209-216.

Merrill JE, Benveniste EN. Cytokines in inflammatory brain lesions: helpful and harmful. Trends in Neurosciences. 1996. 19: 331 -338 .
Park TK, Koppula S, Kim MS, Jung SH, Kang H. Anti-neuroinflammatory effects of Houttuynia cordata extract on LPSstimulated BV-2 microglia. Tropical Journal of Pharmaceutical Research. 2013. 12: 523-528.

Perry RT, Collins JS, Wiener H, Acton R, Go RC. The role of TNF and its receptors in Alzheimer's disease. Neurobiology of Aging. 2001. 22: 873-883.

Uttara B, Singh AV, Zamboni P, Mahajan RT. Oxidative stress and neurodegenerative diseases: a review of upstream and downstream antioxidant therapeutic options. Current Neuropharmacology. 2009. 7: 65-74.

Velasco-Lezama R, Tapia-Aguilar R, Roman-Ramos R, Vega-Avila E, Perez-Gutierrez MS. Effect of Plantago Major on cell proliferation in vitro. Journal of Ethnopharmacology. 2006. 103: $36-42$.

https://doi.org/10.15616/BSL.2017.23.4.411

Cite this article as: $\mathrm{H}$ Kang. Anti-neuroinflammatory Effect of Plantago Major var. Japonica in BV-2 Microglial Cells. Biomedical Science Letters. 2017. 23: 411-415. 